\title{
Descripción clínica de una cohorte de pacientes colombianos con leucemia mieloide aguda
}

\author{
- María Isabel Hernández Estrada, Julián A. Machado, Jeanette Prada Arismendy, Erwing Castillo
}

Instituto Tecnológico Metropolitano, Hospital Manuel Uribe Ángel (Medellín, Antioquia)

Introducción: la leucemia mieloide aguda (LMA) es un tipo de neoplasia que afecta el desarrollo del linaje mieloide en la médula ósea, alterando su capacidad de maduración. Según Globocan 2012, las leucemias son la octava causa de cáncer en Colombia, con una incidencia de 2.628 casos por alto, un ASR de 5,8 y una mortalidad de 4,1. Sin embargo, no se conocen datos específicos sobre $A M L$, lo que genera serias limitaciones para determinar su comportamiento en esta población. En Colombia, los registros publicados sobre LMA en adultos son escasos, y en algunos casos carecen de la información clínica necesaria para realizar su completa clasificación según subtipo y riesgo. Este trabajo describe las características clínicas de una cohorte de pacientes con LMA diagnosticados en un hospital de Colombia.

Materiales y métodos: se revisaron las historias clínicas de pacientes adultos diagnosticados con LMA desde febrero de 2013 hasta diciembre de 2017. Se incluyeron 45 pacientes, de los cuales se recolectaron datos demográficos, clínicos y de laboratorio. Para el análisis de datos, se usa el software estadístico R-Studio.

Resultados: se registró un total de 45 pacientes $(64,4 \% \mathrm{~F} / 35,6 \% \mathrm{M}$, mediana de edad en el momento del diagnóstico 50 altos, rango 18 a 84). El recuento promedio de leucocitos fue de 24.729 células/ $\mu \mathrm{l}$, el recuento de plaquetas 102.444 células/ $\mu \mathrm{l}$ y la hemoglobina $8,72 \mathrm{mg} / \mathrm{dl}$. Al momento del diagnóstico, los blastocitos promedio en la médula ósea fueron del 63,84\%. El tipo más frecuente de LMA fue M3 (clasificación FAB) y la alteración citogenética con mayor incidencia fue la t $(15 ; 17)$. Durante el protocolo de inducción 2 pacientes murieron y otros 9 fallecieron después del tratamiento de inducción o en la fase de consolidación $(25,5 \%)$. Treinta y cuatro pacientes $(74,4 \%)$ permanecieron vivos durante el período de observación. En cuanto a las complicaciones luego del tratamiento de inducción, 38 pacientes (88,37\%) presentaron neutropenia febril; 10 (23,25\%), sepsis; y 8 (18,60\%), otras complicaciones (hemorragia cerebrovascular, anemia y trombocitopenia).

Conclusiones: este trabajo describe la clínica, citogenética y los resultados de pacientes con LMA en un hospital colombiano. Curiosamente, hubo una inversión de la relación mujer:hombre (1,8:1) en comparación con otros informes en la literatura. La leucemia promielocítica aguda fue la leucemia más frecuente, así como la t (15;17), similar a otros reportes latinoamericanos. 